\title{
MICRO AND MACRO ECONOMIC ANALYSIS OF CLOUD COMPUTING
}

\author{
Joseph Packy Laverty, Robert Morris University, laverty@rmu.edu \\ David F.Wood, Robert Morris University,wood@rmu.edu \\ John Turchek, Robert Morris University, turchek@rmu.edu

\section{ABSTRACT}

A 2012 Forester Consulting survey reported that both IT and business managers have been struggling with the complexity and differing perspectives concerning cloud computing. Business leaders CIOs and IT professionals often make decisions concerning choices on architectures infrastructures, platforms, and frameworks based on costbenefit, capital budgeting or business policy decision-making models. While there has considerable research into cloud technologies, there has been less research into the short term and long term economic effects of implementing cloud technologies within the organization, industry or economy. This paper studies the potentials of Micro and Marco Economic analysis to provide better information for cloud computing technology policies

Keywords: Cloud Computing, Decision Making Models, Microeconomics, Macroeconomics, Components of Cloud Computing Technologies, IT Policies

\section{INTRODUCTION}

In its most, simplest form, economics provides types of decision-making models. This paper studies the potentials of a Micro and Marco Economic analysis to provide better information for cloud computing technology policies. Its purpose is to frame the technical decisions and options faced by senior IT executives from an economic perspective, illustrating how the features of cloud computing can be viewed as economic decisions.

Policies, research and analysis of cloud computing has had many threads. These have included 1) an appropriate definition of cloud computing, 2) technological components of cloud computing architectures and services, and 3) identification of business and technology advantages and disadvantages of private and public clouds.

In 2012, Forester Consulting conducted a survey for BMC, Inc. concerning the adoption of cloud computing [7]. While the overall conclusion of the study indicated that $81 \%$ of CIOs surveyed indicated that a comprehensive cloud policy is a high priority, both IT and business managers have been struggling with the complexity and differing perspectives. For example, CIOs are concerned that business leaders see cloud computing as a way to circumvent IT, but agree that a move to cloud computing cannot be stopped. $58 \%$ of the respondents report that mission-critical workloads have been moved to unmanaged public clouds without appropriate policy which caused an increased in IT complexity that may have offset potential cloud benefits.

Business leaders CIOs and IT professionals often make decisions concerning choices on architectures, infrastructures, platforms, and frameworks based on cost-benefit, capital budgeting or business policy decision making models. While there has considerable research into cloud technologies, there has been less research into the short term and long term economic effects of implementing cloud technologies within the organization, industry or economy.

\section{BACKGROUND, DEFINITION AND SERVICES OF CLOUD COMPUTING}

For decades, we have been accustomed to the definition of the "cloud" as being a metaphor for the Internet. Over time the "cloud" evolved into an architecture that shared computer resources using technologies such as timesharing, distributed or grid computing

Many data centers often utilized IT resources at a rate of less than $10 \%$.[14] There were many reasons for this underutilization, e.g., economies of scale, meeting peak period demand, backup capacity, etc., required extra capacity. In 1960, John McCarthy (of artificial intelligence fame) expressed his opinion that computing could be viewed as a "service", which could distribute this excess capacity similar to any public utility company.[25] It was Amazon's vision in 2006 to become an IT utility company to serve large businesses. IBM SmartCloud and Oracle Cloud frameworks followed Amazon's lead and introduced proprietary cloud computing services in late 2011.

Building on the previous success of free Gmail service, Google and other cloud providers introduced more sophisticated cloud applications in 2010. Cloud Multitenancy refers to ability to shared applications and middleware in a cloud computing architecture. Google's strategy was to serve small businesses, education and consumers through a variety of low-cost or free, cloud-based applications and storage. But, Google does not provide VM 
provisioning services such as Amazon. Windows Azure and iCloud later followed adding cloud services to support their proprietary product lines.

There is no universal agreement on the definition of cloud computing. To conduct a micro and macro analysis of cloud computing one must first agree on some operational definitions and boundaries. For the purpose of this analysis, cloud computing is defined by following 1) the commoditization of private or public, distributedvirtualized IT resources at various service levels, 2) designed to take advantage of economies of scale, dynamic scalability, and with low barriers of entry, and 3) where Information and Communication Technology (ICT) costs may be allocated, charged, and shared using either a service-level or pay-per-use model governed by terms set by Service Level Agreements (SLA) $[11,1,13,8,9,4,12]$. Statistics concerning the diffusion of private cloud may be under estimated since many virtualization managers incorrectly classify their architectures and private clouds [12. p2]

Cloud computing services are often delivered to organizations and end-users by reference to popular service levels. Infrastructure as a Service (IaaS) may be classified as renting or usage-based pricing of virtual machines, processing cores, memory, storage, and network bandwidth. Renting a resource involves paying a negotiated cost to have access to a resource over some time period, whether or not you use the resource. Usage-based pricing is not renting. Pay-as-you- go involves metering usage and charging based on actual use, independently of the time period over which the usage occurs.

Platform as a Service (PaaS) may be classified as renting operating systems and middleware. PaaS is likely to be an extension of IaaS.

Software as a Service (SaaS) may be classified as renting applications, web sites, and application database content. Financial, Cost and International Accounting, Sales, Desktop, Enterprise Resource Planning (ERP), Supply Chain Management (SCM), Customer Resource Management (CRM), Human Resources, Recruiting, Payroll, Transportation and Warehouse management applications system are a selection of SaaS applications that may rented using a single-tenancy or multiple-tenancy model $[11,8,15]$.

Single-tenancy architectures provide separate, multiple application instances (or hardware systems) where one instance serves one client or tenant [19]. Multitenancy refers to a principle in software architecture where a single instance of the software runs on a server, serving multiple client-organizations (tenants). The software application must be designed to virtually partition its application data and configuration, such that each client organization works with a customized virtual application instance $[19,17]$.

While IaaS, PaaS, and SaaS are the most frequently cited service levels, the 'as-a-Service' tag has evolved as into an IT marketing tool designed to offer more granular cloud-based services. For example, Security as a Service (SaaS) integrates security services, e.g., authentication, anti-virus, anti-malware/spyware, intrusion detection, and security event management, etc., into a corporate infrastructure on a subscription basis [23].

\section{COMPONENTS OF CLOUD COMPUTING TECHNOLOGIES}

Cloud computing technological structures vary considerably. These differences may affect microeconomic and macroeconomic analysis. Three cloud technology resources are considered: 1) operating system virtualization (virtual machines). 2) computing clusters, and 3) storage virtualization.

By using virtualization technology, a cloud computing provider could provide virtual machines (VMs) and storage capacity on-demand, and in real-time. A mainframe or server often has excess capacity. A virtual machine is an independent, software computer that executes within a larger physical computer. Each VM can support any operating system, file system, and applications. Hundreds of simultaneously, executing VMs are managed by a hypervisor program. Many VMs (software computers) can be dynamically clustered together to share processing of a common application.

Virtualization technology is the key component that enables cloud platform computing. Server Virtualization may provide benefits to reduce physical space, electrical power, environment control, utilization, reduced storage and management. But, sharing of processing resources can create unpredictable performance problems due to the lack of dynamic workload management services. [4]

IBM (1972) was the first to pioneer operating system virtualization. The IBM zEnterprise platform is the only example of hardware-based, operating system virtualization. LPARs, i.e., Logical Partitions, provide greater 
security and performance as compared to any software-virtualization alternatives. However, the number of virtual LPAR images is limited and only supports IBM zEnterprise operating systems.

IBM's zVM (1972) is a software-based virtualization and uses a Type-1 hypervisor similar to VMWare vSphere, Microsoft Hyper-V and Xen. However, it does not directly support Windows Server operating systems, middleware, and desktops at the time of this writing. IBM introduced a new family of Microsoft Server virtualization products in the summer of 2013. However, these IBM virtualization products were only supported by the Blade and System X servers, and did not support zEnterprise mainframes, the king of scalability and performance.

The definition of Type-1 Hypervisors in literature is often described as "bare metal" or hardware-based. There is a significant difference between a virtualization that uses a processor's instruction set to supports virtualization, as compared to virtualization that uses both the physical processor and hardware's firmware to manage virtualization directly. For example, a Type-1 Hypervisor can be hacked, but IBM, LPAR firmware virtualization is almost impervious to compromise.

Private or Public Cluster technologies permit workloads to be distributed over multiple physical computers, LPARs, processing cores, or virtual machines. Depending on the configuration of the guest operating system, middleware, or application, workloads may be distributed in a symmetric (parallel processing of multiple threads) or asymmetric processing mode.

High-performance clusters (SMP) use cluster nodes to perform concurrent calculations. One task is shared simultaneously by multiple processing units. Load-balancing clusters (AMP) dispatch network service requests to multiple cluster nodes to balance the request load among the cluster nodes. Load balancing provides cost-effective scalability because you can match the number of nodes according to load requirements. One task is assigned to one processor unit and the processing requirements are not shared.

High-availability clusters provide continuous availability of services by eliminating single points of failure and by fail-over services from one cluster node to another in case a node becomes inoperative. Storage clusters provide a consistent file system image across servers in a cluster, allowing the servers to simultaneously read and write to a single shared file system.

Not all high-performance (parallel) clusters are the same. In order to coordinate and manage processing integrity between multiple parallel processors, there will be a significant processing overhead in most cluster technologies. For example, the performance of one processor clustered with a second processor, virtual machine, or sever is not equal to 2 , i.e., $1+1$ does not equal 2 . Rather $1+1$ may equal 1.9 , or $1+1+1$ may equal to 2.6 , and so forth.

The reduction of marginal net output (Marginal Physical Product) is due to the increased demand for cluster process coordination and integrity overhead. In economics, this is called the Law of Diminishing Returns. In fact, a cluster of more than eight UNIX/Linux servers will experience "negative", not diminishing returns for most workloads, i.e., $8+1=7.7$. Windows Servers will also experience negative returns after the addition of the seventh server. But, IBM's z/OS Parallel Sysplex servers will not even experience diminishing returns until the 33rd server is added to the cluster. IBM will not permit a cluster of more than $32 \mathrm{z} / \mathrm{OS}$ servers. While IBM zEnterprise server Parallel Sysplex technologies are expensive, no other alternative clustering technology comes close to scalability, performance or reliability of an IBM parallel cluster.

Load-Balancing clusters operate differently from High-Performance clusters. The coordination overhead occurs between tasks, not within a task. The point of diminishing returns or negative returns varies by task, platforms and cluster algorithm. However, the ability to extend load-balancing cluster sizes to 1,000 of servers is an exaggeration. In addition, the processing capabilities of one IBM EC12 mainframe are approximately equal to 86 Windows 2012 multiprocessor/multicore servers. While the Windows 2012 Server can theoretically support up to 64 processors, there are no motherboards capable of supporting this number. The Intel server processor speed is almost $1 / 3$ of one IBM EC12 processor and has limited support processor coupling necessary for specialized cluster operations.

One advantage of cloud computing is the rapid provision of additional processing and storage resources to a task. Rapid provisioning from VM images may be faster than traditional installation methods, but does not address the need for dynamic workload management. As the name implies dynamic workload management will add virtualization or hardware resources both dynamically and automatically (immediately) based on some preset performance policy or agreement. 
The technical difference does not normally matter with SMEs (small and medium size enterprises) with a single workload, e.g., maintaining a web application website. However, when the scale of the requirements varies and the workload increases, dynamic workload management becomes extremely important for large enterprises.

To imply that public cloud architectures fits-all-sizes, variability and workloads in a cost-efficient manner is a distortion typically made by technologies in their infancy. Just consider the performance of Obama Care's web site rollout. Consider that the transaction processing and storage capacity of any one of the top Fortune 100 companies exceeds the total of the top five cloud service providers in 2012. Neither do these points imply that there may not be a role for both private and public clouds for any scale of enterprise. The benefits of cloud services are not a continuous function.

Storage Area Network (SAN) Technologies provide opportunities for increased storage utilization, capacity for variable storage, and shared costs in private and public cloud technologies. Many organizations double storage capacity each year for transaction, data warehousing, and government compliance. Reduced provisioning time to expand storage, centralized data storage management, fault tolerant 24/7 reliability, and improved backup and data recovery are often cited technological advantages. No SAN technological review would be complete, without consideration of "data lock-in problems dues to the proprietary nature of SANs $[9,12]$, increased communication bottlenecks and security risk due to centralization of data, and availability of qualified storage administrators.. While most cloud administrators have server/virtual service experience, less than $30 \%$ have storage administration experience [12, p2].

\section{FOUNDATIONS OF MICROECONOMIC AND MACROECONOMIC ANALYSIS}

Technology and business analyses are important in the selection and implementation of ICT. These analyses will be used to start microeconomic and macroeconomic analysis (MMEA). MMEA will analyze additional classifications of costs and benefits, such as, opportunity costs, sunk cost, fixed/variable costs, sensitivity factors, e.g., price, income and substitutions, differences between time frames, and effects of economic growth, employment, and government policy.

Microeconomics is the study of decisions that consumers, businesses, and industries make regarding the allocation of resources and prices of goods and services. Microeconomic analysis often begins with an analysis of demand and supply. For example, computing architectures may have a significant effect of reducing the marginal cost-per-unit (MC) of technology, which will affect resource and product pricing. In turn, cloud technologies may lower equilibrium prices for products and services to consumers, businesses and governments.

Cloud technology may have several cost advantages, but consumers must be willing and able to purchase goods and services. Therefore, the demand curve is as important as the supply curve. Some economists often describe the demand curve as a monetized, declining marginal utility curve. A utility, or until, is a unit of satisfaction.

Why does one purchase wine? First, we purchase wine because it satisfies us. Second, are we as satisfied with our fifth glass of wine as our first? No. This concept is called Declining Marginal Utility, which means that to encourage more consumption of any product or productive resource, prices must fall. But, if the product or service is not valued at all, e.g., has no utility to the buyer, it may not matter how low the price may fall due to lower technology or production costs..

Demand elasticity analyzes the effects of a change in prices, change income condition of the buyers, or comparable prices of substitutes and complimentary products. Elastic Demand (Sensitive) means consumers, business and governments will significantly increase quantity demanded for decreases of market prices, increase in prices of substitutes, or an increases in income of a buyer. Inelastic Demand (Insensitive) means consumers, business and governments will not significantly increase quantity demanded for decreases in market prices, increases in prices of a substitute, or increases in income of a buyer.

The size of the effect of changes in technology costs is also dependent on demand elasticity. Lowering production or technology costs may lower consumer prices, buy how big is the effect? Demand Elasticity determines "how much" does "more" mean?

The demand for consumer technologies are at an all-time high; whereas, the demand for business and government technologies are declining. Without amending the U.S. Constitution, it is now assumed that owning a personal cell phone or flat panel TV are inalienable rights. In 2014, Apple was ranked 19th as Global Fortune 500 Companies, while IBM had fallen to $62 \mathrm{nd}$. 
The implementation of cloud computing architectures may affect the out-of-pocket technology costs, thereby, shifting the supply curve to the right and outwards. This MAY lower the technology Total Cost of Ownership, but in the bigger picture but these changes may have no effect on the product and service prices or quantity demanded for some industries and first.

While some may cite that cloud computing may decrease the barriers to entry or risks to innovate, consider the law of declining marginal utility. Increasing the use of anything lowers the perceived value. For example, many consumer feel that Internet access, services, mobile apps should be at no-cost. Consumer cloud storage is free up to 15-gigabytes for many cloud and communication technology providers. While cloud analysis has focused on the cost-side, commoditization and excessively lower costs introduce by cloud computing may make it increasingly more difficult for businesses to charge for any technology. Simply consider free checking and ATM machine expectations. Supply or demand curve shifts may provide for an economic profit in the short-run, but depending on elasticity may or may not be sustained.

Macroeconomics is the study of economics of an industry; private versus public sectors, regional, national and global economies; and regional, national, and global government policies. For example, Federico Etro (2011) [11] projected that as a result of the diffusion of cloud computing the manufacturing sector would benefit the most when measuring net job growth (non-ICT jobs created less ICT jobs lost). However, other industries would be affected by a decrease in net job growth, e.g., retail, wholesale, real estate, finance, hotel and other businesses. Net job-growth is expected to vary by country [23].

Besides the effects on job creation and destruction, the diffusion of cloud computing may affect the growth of GNP, barriers to entry, industrial concentration, inflation, the cost of government services and tax revenues, and international comparative advantages [23].

\section{MICROECONOMIC ANALYSIS OF CLOUD COMPUTING}

\section{Conversion of up-front, committed fixed costs to variable discretionary costs}

ICT up-front costs are often evaluated using capital budget models, e.g., net present value. Cloud-based architectures offer the opportunity to convert fixed cost into periodic, variable or mixed costs. Cloud-based capital budgeting models should treat the cloud computing similar to buy-or-lease, while incorporating differential financing, tax effects, intangible and other economic costs [11,14].

Lower break-even points will reduce operational and business risks. Flexible and dynamic provisioning will provide opportunities technology-based industries, e.g., banking, insurance, investment, and finance. On the other hand, the same factors may also increase economic concentration in those industries.

\section{Purchasing economies of scale for SMEs}

Harms and Yamartino (2012) has estimated that small-to-medium enterprises (SMEs) may enjoy 20-30\% savings due to purchasing economies of scale provided to Cloud Service Providers (CSP). Is it plausible that CSPs will pass all of these saving to the customer? $[13,14]$

\section{Operational economies by balancing different utilization patterns}

Cost saving benefits may be possible by balancing different utilization patterns by a) time-of-day, b) time zone, c) industry-specific variability (retail Christmas sales versus tax services), d) application (search versus email tasks), e) workload (batch versus OLTP), and f) uncertain growth patterns [14].

\section{Reduction of electric utility expenses}

Electricity costs have been rapidly increased represent $15-20 \%$ of total operational costs. Power Usage Effectiveness (PUE) tends to be significantly higher in larger data centers than small data centers due to geographical variability, economies of scale, and negotiated rate reductions [14].

\section{Reduction of physical data center space}

In additional to basic construction costs, data centers often require additional special structural, environmental, redundancy and security requirements. The possibility to reduce data center space requirements may be significant for either private or public cloud architectures. 
The potential to re-purpose the data center floor space, however, may be limited for private cloud architectures. The security and structural constraints may prevent re-assignment of a data center's floor space to an alternative function. Renting or consolidating data center space with other companies ICT needs may be a possibility.

Existing data center space may be a significant fixed cost pool, but it also may represent a sunk cost. Sunk costs are a subcategory of fixed costs, which are not differential and should not affect future economic decisions. For example, the choice to outsource data center operations to a public cloud service provide should not be consider data center physical costs under most conditions. It does not matter if the existing data center operational model is retained or outsourced to a public cloud service provider. There would be no difference in allocated depreciation expense or capital write-offs.

If the differential or incremental benefits exceed the differential or incremental costs, it does not matter what amount of depreciation is allocated from an economic model's perspective. The decision is to accept the proposed change. Allocated costs are not normally not differential, such as depreciation and amortization expenses. Data center structural depreciation is normally a sunk cost unless one can re-purpose the facility. Estimates concerning footprint savings should be carefully analyzed.

\section{Reduction of ICT labor costs}

Some ICT Labor Costs in cloud architecture decisions may decrease, but should be carefully analyzed to determine if these costs are differential. Microeconomic resource decision models state that the relative combination of capital and labor will be optimal where the ratio of the Marginal Physical Product of Capital (MPPC) relative to its Marginal Resource Cost of Capital (MRCC) is equal to Marginal Physical Product of Labor (MPPL) relative to its Marginal Resource Cost of Labor (MRCL).

Since cloud computing would be expected to lower the marginal resource cost of technology capital, one would expect that ITC labor utilization will be reduced under normal circumstances because cloud computing became a less expensive capital cost. But, the actual amount of the reduction would be determined by the substitution effect between resources. How much would ITC labor costs be reduced may vary.

Resource substitution effects are also determined by resource sensitivity factors; such as the ability to replace ITC labor by capital and the percentage of ITC labor costs in the relationship to the total IT budget. There may be labor cost savings which results operational operating system and application updates, server and application provisioning, operation monitoring, backup and recovery costs. But, other ICT jobs and costs may be actually un affected.

In addition to IT capital/labor productivity ratio analysis, one should consider the effects of raising health care and retirement costs throughout the organization. Cloud computing may provide the opportunity to management for ITC labor to share in the pain. From a microeconomic point of view reduction of ITC labor and costs will be good. From a macroeconomic point of view, the potential for increased unemployment may undesirable.

Some ICT jobs may be actually re-assigned within the ICT department. When ICT personnel are limited, i.e., a constraint, the "opportunity costs" to assign IT personnel to other roles and projects may be significant. As cloud computing releases IT personnel constraints, opportunity costs are lower and new IT projects may now become feasible.

The labor savings as the result of cloud computing benefits should be reduced by any retraining costs. Some ICT jobs lost may be actually re-assigned outside the ICT department to a lower value activity, which has been a popular policy in the public sector. It is important to correctly identify the net labor savings attributed to microeconomic benefits of cloud computing.

\section{Consolidation and shared use of processing and storage resources [6,9] -- Tangible and Intangible Costs and Benefits of Multitenant Applications}

An application instance usually incurs a certain amount of memory and processing overhead, which can be substantial when multiplied by many customers. Multitenant applications and middleware simplifies the upgrade and new release management processes and may reduce these some types of differential overhead costs by amortizing it over many customers [19,17]. Multitenant Applications may permit greater economies of data aggregation and potentials for data mining. Instead of storing transactional and decision support data in multiple data stores, with a potential of different database schemas, all data for all multitenant users is stored in a single database schema. 
The benefits of multitenant applications and middleware may be limited by the availability and extra cost of multitenant licenses, scalability, and increased security threats and vulnerabilities, customization complexity and maintenance of per-tenant metadata. Furthermore, multitenant applications and middleware may also increase complexity and risk of an unsuccessful upgrade or new release, longer downtimes, and the coordination of user training between multiple tenants.

\section{Intangible benefits of cloud security for SMEs}

Operational security hardware and expertise costs are normally fixed costs. Increasing the number of users and organization applications will decrease security-related costs per cloud user or organization and provide security expertise that would not be available otherwise. Intangible benefits include the increased a) quality of security planning, b) security testing, and c) responsiveness to a security event.

External verification of cloud service provider's security claims is only acceptable as can be externally verified. The quality of cloud security verification standards vary and are in their infancy. For example, the SAS70 provides limited assurance. Organization, such as the National Institute of Standards and Technology (NIST), General Service Administration (GSA), Cloud Security Alliance (CSA), the Federal CIO Council, and the European Network and Information Security Agency (ENISA) have proposed standards to verify and audit cloud service providers which vary considerably[20].

\section{Intangible costs of cloud security for SMEs}

Private and Public Cloud architectures also increase the types and complexity of security vulnerabilities. For example if a hacker can compromise the hypervisor layer, they can avoid detection by most security software running at the application layer, will have greater access to the system and the possibility that they will be able to remotely control all virtual machines and storage area networks. Organizations have less control over public clouds. Proprietary extra-chassis links and protocols to enable cluster technologies present additional concerns [20].

Hackers have always exploited a system's biggest shortcoming, one that is virtually impossible to secure -the human user. The use of a single password and user ID across all of a computer user's system interactions, or the use of trivial user IDs and passwords, are well-known exploits that can provide weakly defended backdoors into higher security components. The increased benefits of cloud-based security may not directly address batch, OLTP, and web application, operating system security and middleware vulnerabilities

\section{Other intangible costs of cloud computing}

Cloud computing has increased risks concerning 1) the business continuity of the cloud provider, 2) the intrinsic nature of the cloud business model, 3) erosion of control and accountability, 4)potential CSP outsourcing, 5) additional subpoena and e-discovery risks, 6) insider threats, 7) loss of governance, 8) unauthorized physical access as CSPs, 9) media destruction, 10) service isolation, and 11) privacy concerns $[23,20]$.

\section{MACROECONOMIC ANALYSIS OF CLOUD COMPUTING}

\section{Lower Barriers to Entry and Innovation Effects}

The effects of decreasing the fixed cost of ICT may lower the barriers to entry in non-ICT sectors; thereby increasing competition, lowering prices and encouraging innovation [11]. This premise suggests that cloud computing systems would enable SMEs (Small and Medium Enterprises) to compete with the "big-boys."

The potential of cloud computing technologies to lower barriers to entry may be attributed to 1) pay-as-you-go pricing, 2) ability leverage economies of scale, 3) rapid provisioning, 4) future dynamic scalability, and 5) availability to easy-to-use cloud tools and cloud support specialist. For example, a SME can simply contact Amazon Cloud Services to provision a VM, with any operating system or web technologies, applications, storage and network bandwidth in a matter minutes. The costs will be low, discretionary and variable and the ICT resources will be scalable to the requirements of the business model.

The ability to enter an industry will vary. SMEs may be faced with other non-technological barriers to entry, e.g., flame-broiled burgers are always better than fried. Existing brand-name identification, product differentiation, patents and copyrights may be challenging to overcome. There is also a question whether a SME will have the expertise to develop an innovation or the budget to market the innovation. Many great web sites and social 
networking strategies have failed to attract visitors. Availability of low cost ITC resources does not guarantee successful application of innovations.

However, some SMEs have had a record of successful innovations, especially those that require significant computing power to process calculations or complex simulations and testing tasks. Information to support organizational decision making is also an asset. Google provides user free services just for the right to collect user data. Collecting transactional data is not very expensive. On the other hand, data analysis and data mining requires significant processing and storage resources which cloud computing may be available to SMEs at a reasonable or no cost through cloud-based computing clusters.

AWS provides no-cost cloud services through their college and university academic grants. Most of these grants have been awarded for basic research and data mining projects. Cloud computing puts a supercomputer into the hands of every developer, enables users to experiment often and fail quickly, and enables big data without big servers

On a sarcastic note, the benefits of SME cloud-based innovation may be limited in the long-run. What happens to innovative SMEs? Many are eventually "gobbled-up" by the big-boys in the long run.

\section{Potential to increase production and employment in non-ICT Industries - secondary spending effects}

Increased competition, innovation, and lower ICT costs may cause increased production and employment opportunities in non-ICT economic sectors. Increased employment effects will be limited by industry. Federico Etro (2011) stated that manufacturing, retail, wholesale, real estate and finance employment and output are expected to benefit more than other sectors. However, the long-term impact on wage levels in these sectors may be insignificant. The transportation, communications, hotel and restaurant sectors will be affected only slightly. But, consumption multiplier effect will definitely increase GNP and employment. However, the type of employment and output effect may also differ by country [11].

The capital/labor resource substitution-effect will also determine the direction and level of expected employment changes. Marginal Resource Cost (MRC) is only one of the factors that determine labor and capital utilization. The relative Marginal Physical Product (MPP) will also affect the relative utilization of labor and capital resources. For example, no matter how cloud computing lowers ICT costs or capital innovations are introduced in the surgery room, demand for the number of surgeons will not dramatically change by technology. You cannot substitute capital for the labor cost of a surgeon. On the other hand, McDonalds has replaced employee watching the French Fryer employee, with that timer and buzzer. (Would someone please shut that buzzer off?)

The faster the rate of diffusion of cloud computing for SMEs, the greater the impact will be on non-ICT output and employment. But, these output and employment increases are expected to be negligible at best, e.g., $0.1 \%$ to $0.4 \%$ [11].

\section{Potential for lower prices, markups and inflation in the long-run}

Federico Etro [11] predicted that the impact of cloud computing may lower prices, markups and inflation. Etro's macroeconomic projections assume that an inelastic demand curve for industries which may be able to take advantage of cloud computing. A reduction of ITC costs will shift the supply curve downwards and to the right. The shift will change the demand-supply equilibrium point.

The magnitude of the price changes are partially determined by the price elasticity of the demand curve. Price elasticity measures the sensitivity of prices and outputs to changes in price. Some production sectors and long-run demand curves are assumed to have an elastic demand. An increase in supply (lower costs of production) may result in more production and employment in the short run, but may not be reasonable in other industries or in the long run. The bottom line is that the premise the lower costs of cloud computing architecture will lower product and service prices or inflation is highly questionable.

\section{Public sector use of cloud computing may decrease government spending.}

In September 2009, the Obama Administration introduced the FCCI (Federal Cloud Computing Initiative), with the purpose of promoting standards and rules for the adoption of cloud services to reduce the $\$ 76$ billion of ITC 
spending for the American government. In Europe the U.K. is trying to convert traditional IT architectures to cloud computing at a rate of $10 \%$ a year to reduce the $£ 16$ billion ITC spending by the British government $[11,17]$.

It is difficult to project the value of microeconomic or macroeconomic benefits of cloud computing in either private or public sectors due to the "endogeneity problem". An endogenous variable effect may occur when one attempts to project benefits of cloud computing when other outside variables may create a greater distortion than measuring the relationship between independent and dependent variables.

The basic assumption of capitalism and the private sector is that businesses will attempt to "maximize profits" by either increasing demand or lowering costs of supply. If a business fails in the private sector they will go out of business.

However, the basic assumption of the public sector is to "maximize votes." Public sectors never go out of business. Some lower-level government bodies may go bankrupt, but often will simply pass on the loss to another government body. The public sector has little incentive to efficiently use or manage labor, capital or computer resources. Has government labor costs ever decreased? Has government purchased any major item based on cost or efficiency, or are they motivated to give the contract to the biggest campaign donor? The role of government is an endogenous variable, which distort the predictions of cloud computing benefits.

\section{Increased non-ICT employment may increase tax revenues.}

Depending on the redistribution policy embedded in tax policy, i.e., the low income tax credit, tax revenues should expect to be increased by cloud computing [11]. The introduction of the personal computer into the 1980s and 1990s had significant impact on the stock market and government tax revenues. For those Democratic or Republican political supporters out there, the introduction of the personal computer had more impact than the policies of either Bill Clinton or Ronald Reagan. But, it is doubtful that diffusion of cloud computing will have any major impact.

\section{CONCLUSIONS AND RECOMMENDATIONS}

This study considered the use of microeconomic and macroeconomic analysis to improve business and IT policies, and to improved decisions concerning cloud computing technologies. First, economic analysis is designed to consider costs and benefits not normally considered in financial, capital budgeting decision-making models or analysis. Intangible costs or benefits security, innovation, resource opportunity costs, business continuity of the cloud provider, erosion of control and accountability, multitenant applications, CSP's outsourcing, additional subpoena and e-discovery risks, insider threats, governance loss, unauthorized physical access, media destruction, service isolation and privacy should be considered.

Second, economic analysis properly evaluates non-differential costs and benefits, sunk costs, and allocated costs as compared to many other decision-making models and policies. Allocated costs such as depreciation and amortization expenses are normally not differential. Business managers and accountants may rely on a full-cost analysis that may not be appropriate.

Finally, economic analysis forces decision makers to consider external factors, e.g., economic employment, growth, inflation, and other variables in both the short run and the long run. Technology changes and opportunities affect more variables than the individual organization. Economic analysis forces business to evaluate technology changes beyond the internal policy.

The conclusion of this paper is that microeconomic and macroeconomic analyses are recommended decision-making tools to supplement any IT technology policy, no matter the technological complexity. It forces he decision maker to view the technological "forest" in the entire economy, as well as, each individual technology "tree".

\section{REFERENCES}

1. Adekunle, Y.A, Maitanmi, S.O, Malasowe, B.O, Osundina, S. A, (2012). Economics of Cloud Computing. International Journal of Engineering and Innovative Technology (IJEIT), 1(4).

2. Amazon Elastic Compute Cloud (Amazon EC2). (2014). Available: http://aws.amazon.com/ec2/\#pricing

3. Amazon S3 Pricing. (2013). Available: http://aws.amazon.com/s3/pricing/

4. Armbrust, M. Armando, F. Griffith, R., Joseph, A., Katz, a., Lee, G, Patterson, D., Rabkin, A. Stoic, A., and Zaharia, M. (2010). A View of Cloud Computing. Communications of the ACM, 53(4). 
5. AWS Economics Center. (2014). Available: http://aws.amazon.com/economics/.

6. Banerjee, U. (2012). Gartner Outlines 5 Cloud Computing Trends - What They Really Mean. Available: http://setandbma.wordpress.com/2012/04/24/gartner-5-cloud-trends/

7. BMC Software Survey Signals Comprehensive Cloud Strategy is Needed to Alleviate Growing Tensions between Business and IT Teams. (2012). Available: http://www.bmc.com/news/pressreleases/2012/BMC-Software-Survey-Signals-Comprehensive-Cloud-Strategy-is-Needed.html

8. Chou's Theories of Cloud Computing: The 5-3-2 Principle-. (2013). Available: http://blogs.technet.com/b/yungchou/archive/2011/03/03/chou-s-theories-of-cloud-computing-the-5-3-2principle.aspx

9. Cisco IT Attains $140+\%$ Storage Utilization, Realizing Over $\$ 8$ Million in TCO Savings with NetApp Storage. (2013). Available: http://www.netapp.com/us/system/pdf-reader.aspx?pdfuri=tcm:10-60066$16 \& \mathrm{~m}=$ cisco.pdf

10. Cloud Security Alliance Security as a Service. (2014). Available: https://cloudsecurityalliance.org/research/secaas/\#_overview

11. Entro, F. (2009). The Economic Impact of Cloud Computing on Business Creation, Employment and Output in the E.U. Review of Business and Economics, 54(2). 179-208.

12. Forrester Consulting. (2011). Private Clouds: Storage Architectures Are Key to Maximizing Efficiencies. Available: http://www.readwriteweb.com/files/Forrester_report_rp-storage-architectures.pdf

13. Gartner, (2009). Dataquest Insight: Many Midsize Businesses Looking Toward 100\% Server Virtualization.

14. Greenberg, A., Hamilton, J., Maltz, D., Patel, Parveen. (2009). The Cost of a Cloud: Research Problems in Data Center Networks. ACM SIGCOMM Computer Communication Review, 68(1).

15. Harms, R. \& Yamartino, M. (2012). The Economics of a Cloud. Available: http://www.microsoft.com/en-us/news/presskits/cloud/docs/the-economics-of-the-cloud.pdf

16. IDC (2008). The Economics of Virtualization: Moving to an Application-based Cost Model.

17. Johnson, D. (2013). Cloud Computing - The Oath to Increased Efficiencies and Cost Savings for Government Agencies. Available: http://www.cristie.co.uk/media/2136/wpcloud_computing_increased_perfromance.pdf

18. Marston, S., Li, Zhi, Bandyopadhyay, S., Zhang, J. \& Ghalsas, A. (2010). iCloud computing -The business perspective. Available: http://ac.els-cdn.com/S0167923610002393/1-s2.0S0167923610002393-main.pdf?_tid=0c95c78c-d7a2-11e3-8bd200000aacb361\&acdnat=1399657854_e4aab0af789401aec13f1bdcff2ea944.

19. Multitenancy. (2014). Available: http://en.wikipedia.org/wiki/Multitenancy

20. Protect your IT systems with next generation security. IBM PurFlex Security. (2013). Available: http://public.dhe.ibm.com/common/ssi/ecm/en/waw12355usen/WAW12355USEN.PDF

21. Security as a Service (SaaS) (2014). Available: http://searchsecurity.techtarget.com/definition/Securityas-a-Service

22. Tak, B., Urganonkar, B, and Sivasubramaniam, A. (2011). To Move or Not to Move: The Economics of Cloud Computing. Available: https://www.usenix.org/legacy/event/hotcloud11/tech/final_files/Tak.pdf

23. Winkler, V., (2011). Securing the Cloud. Syngress, Elsevier, Inc.

24. Wyman, O. (2010). Shared Services Approach Delivers Increased Benefit. Available: http://www.computerworld.com.au/whitepaper/370365/success-story-suncorp-partners-with-netapp-totransform-it-for-competitive-advantage/?type $=$ other\&arg $=0$ \&location=featured_list

25. Yu, H., Powell, N., Stembridge, D., Yuan, X. (2012). Cloud Computing and Security Challenges. Proceedings of the 50th Annual Southeast Regional Conference, ACM-SE '12. 298-302. 